\title{
Tecnura
}

\section{Uso de materiales reciclados en compuestos cementicios}

\section{Using recycled materials in cementitious composites}

\author{
Hernán Xargay ${ }^{1}$, Marianela Ripani², Antonio Caggiano³, Paula Folino ${ }^{4}$, Enzo Martinelli ${ }^{5}$
}

Fecha de recepción: 6 de octubre de 2018

Fecha de aceptación: 14 de febrero de 2019

Cómo citar: Xargay, H., Ripani, M., Caggiano, A., Folino, P. y Martinelli, E. (2019). Uso de materiales reciclados en compuestos cementicios. Tecnura, 23(60), 38-51. DOI: https://doi.org/10.14483/22487638.14697

\section{Resumen}

Contexto: La producción de hormigón se caracteriza por una importante demanda de energía y materias primas, emitiendo grandes cantidades de gases de efecto invernadero (GEI). Asimismo, la construcción, mantenimiento y demolición de edificios genera enormes cuantías de residuos que requieren costosos y ambientalmente sensibles procedimientos de disposición final. Por tanto, en la actualidad se están investigando diversas soluciones para reducir el impacto ambiental de los procesos asociados al ciclo de vida del hormigón.

Metodología: Se estudiaron, mediante ensayos experimentales, las propiedades físicas y mecánicas de los siguientes materiales sustentables: hormigón con agregados reciclados, hormigón con reemplazo parcial de cemento Portland por cenizas volantes y compuestos cementicios reforzados con fibras recicladas.

Resultados: El uso de agregados gruesos reciclados degradó las características mecánicas del hormigón debido a su mayor porosidad y capacidad de absorción de agua. Sin embargo, su combinación con cenizas volantes mostró un efecto sinérgico, mitigando las consecuencias adversas mencionadas. La respuesta posfisuración del hormigón reforzado con fibras de acero recicladas se caracterizó por una menor tenacidad y ductilidad respecto a los compuestos con fibras industriales. Específicamente, las mezclas con fibras recicladas mostraron una etapa de ablandamiento más pronunciada. Esto reveló una eficiencia menor de las fibras recicladas con respecto a las industriales.

Conclusiones: Los resultados experimentales demostraron que la incorporación de materiales reciclados condujo a un deterioro en el comportamiento físico y mecánico-resistente de los compuestos analizados. No obstante, las propiedades resultantes superaron los valores mínimos recomendados para su aplicación como materiales estructurales.

Palabras clave: compuestos cementicios, hormigón, fibras, reciclado, sustentabilidad.

Ingeniero civil, especialista en Ingeniería del Petróleo y Derivados. Docente en LMNI, INTECIN, Facultad de Ingeniería, Universidad de Buenos Aires. Investigador en Departamento ICES, Comisión Nacional de Energía Atómica (CNEA). Buenos Aires, Argentina. Contacto: hxargay@fi.uba.ar ORCID: https://orcid.org/0000-0003-2403-5155

2 Ingeniera civil, doctora en Ingeniería. Docente en LMNI, INTECIN, Facultad de Ingeniería, Universidad de Buenos Aires. Buenos Aires, Argentina. Contacto: mripani@fi.uba.ar ORCID: https://orcid.org/0000-0002-9088-6030

3 Ingeniero civil, doctor en Ingeniería. Docente en LMNI, INTECIN, Facultad de Ingeniería, Universidad de Buenos Aires. Buenos Aires, Argentina. Estudiante posdoctoral en Institut für Werkstoffe im Bauwesen, Technische Universität Darmstadt. Darmstadt, Alemania. Contacto: acaggiano@fi.uba.ar ORCID: https://orcid.org/0000-0003-1027-2520

4 Ingeniera civil, doctora en Ingeniería. Docente en LMNI, INTECIN, Facultad de Ingeniería, Universidad de Buenos Aires. Buenos Aires, Argentina. Contacto: pfolino@fi.uba.ar ORCID: https://orcid.org/0000-0001-5508-0755

5 Ingeniero civil, doctor en Ingeniería. Docente en Università di Salerno, Dipartimento di Ingegneria Civile. Fisciano, Italia. Contacto: e.martinelli@unisa.it ORCID: https://orcid.org/0000-0003-3572-7528 


\section{Abstract}

Context: Concrete production is characterized by a significant demand of energy and raw materials, and by emitting large amounts of greenhouse gases (GHG). Moreover, the processes of construction, maintenance, and demolition of buildings generate huge quantities of waste that require costly and environmentally sensitive disposal procedures. Therefore, several solutions are being investigated to reduce the environmental impact of the processes associated with the life cycle of concrete.

Methodology: Through experimental tests, the physical and mechanical properties of three sustainable concrete mixtures were studied: concrete with recycled aggregates, concrete with partial replacement of Portland cement by fly ash, and cementitious composites reinforced with recycled fibers.

Results: The use of coarse recycled aggregates degraded the mechanical characteristics of the concrete due to their greater porosity and water absorption capacity. However, the mixture made out of recycled aggregates and fly ash showed a synergistic effect, mitigating the adverse consequences mentioned. The post-cracking response of concrete reinforced with recycled steel fibers was characterized by a lower tenacity and ductility compared to composites with industrial fibers. Specifically, mixtures with recycled fibers showed a more pronounced softening stage. This revealed a lower efficiency of recycled fibers compared to industrial fibers.

Conclusions: The experimental results showed that the incorporation of recycled materials led to a deterioration in the physical and mechanical behavior of the analyzed composites. However, the resulting properties exceeded the recommended minimum values required for their application as structural materials.

Keywords: cementitious composites, concrete, fibers, recycling, sustainability.

\section{INTRODUCCIÓN}

Según su definición original y más citada, el desarrollo sostenible debería "satisfacer las necesidades del presente sin comprometer la capacidad de las generaciones futuras de satisfacer sus propias necesidades" (World Commission on Environment and Development, 1987). Por tanto, la industria de la construcción está enfrentando el gran desafío de hacer que sus procesos sean más sustentables. El sector de la construcción de edificios y la fabricación de cemento son responsables de una parte significativa de las emisiones y de la demanda de materias primas a nivel mundial. En efecto, el $40 \%$ de las emisiones globales de GEI antropogénicas y el $40 \%$ de las materias primas se atribuyen a la industria de la construcción, mientras que la producción anual global de hormigón se aproxima a 25 gigatoneladas, es decir, 3,8 toneladas por persona por año (Gursel, Masanet, Horvath y Stadel, 2014). Por otra parte, la generación de residuos de demolición requiere cada vez más capacidad de rellenos de disposición final y esto, especialmente en algunos países, implica un impacto considerable en el medio ambiente (Robayo-Salazar et al., 2015). Debido a las enormes cifras mencionadas, cualquier acción capaz de reducir tanto las emisiones de GEI como la demanda de materias primas puede producir potencialmente un efecto global significativo en el ambiente. En este sentido, reciclar hormigón o reusar residuos de otros orígenes en el hormigón para transformarlos en materias primas secundarias sostenibles son soluciones apropiadas para responder a los requisitos anteriores y lograr una mayor sostenibilidad en la industria de la construcción.

En los últimos años, se han investigado y desarrollado diversas alternativas para la producción de hormigones de bajo carbono (low carbon concrete, LCC), denominación que define a aquellos hormigones elaborados mediante la selección de constituyentes y procedimientos orientados a reducir la huella de carbono y la demanda de materias primas naturales (Villegas-Flores y Parapinski dos Santos, 2013). En este trabajo se estudiarán particularmente los LCC elaborados con: agregados gruesos reciclados, mezclas cementicias que 
contienen adiciones de subproductos industriales y compuestos con fibras de acero provenientes de neumáticos reciclados.

En el hormigón con agregados reciclados (recycled aggregate concrete, RAC) los agregados gruesos naturales son parcial o totalmente sustituidos por agregados reciclados, obtenidos a partir de la selección, trituración y procesamiento del hormigón de desecho. Esta formulación presenta dos grandes ventajas: por un lado, su uso ayuda a preservar las fuentes de agregados naturales y consecuentemente a reducir el impacto ambiental asociado con su explotación, y por otro, permite reducir la cantidad de escombros. Estos últimos provienen de distintas fuentes, entre las que pueden mencionarse: demoliciones, sobrantes de la construcción de obras, y catástrofes, como por ejemplo, las provocadas por sismos de gran magnitud. Las propiedades de los agregados juegan un papel fundamental en el comportamiento de RAC en estado fresco y endurecido, tanto en términos reológicos, de respuesta mecánica, como en su durabilidad a largo plazo. Los agregados reciclados obtenidos a partir de la trituración de hormigón (recycled concrete aggregates, RCA) presentan una mayor porosidad que los agregados naturales debido a que habitualmente una capa del mortero original permanece adherida al agregado reciclado luego del proceso de trituración. Esta capa modifica las propiedades de la zona de transición o interface, con una fuerte incidencia sobre el mecanismo de falla de RAC (Ripani, Etse y Vrech, 2017). Si bien son muchos los factores que inciden sobre las propiedades de RAC, resulta fundamental el porcentaje de reemplazo $(R)$ de agregados naturales. En la actualidad, algunas guías internacionales de diseño tienen en cuenta la utilización de agregados reciclados $(\mathrm{ACl}$ 555-R01, 2001; 121 DRG RILEM TC, 1994). En general, se recomienda limitar a valores bajos el porcentaje de reemplazo de agregados naturales por agregados reciclados (alrededor del $30 \%$ ) y en estas condiciones, se acepta el uso de ese hormigón para fines estructurales. La utilización de RAC en porcentajes bajos de reemplazo ya es una realidad en muchos países, incluyendo a Argentina, a pesar de que los códigos de diseño estructural nacionales no han sido actualizados debidamente para tener en cuenta la incidencia de estos nuevos componentes en el comportamiento global de la mezcla.

Como se mencionara anteriormente, otra alternativa sustentable consiste en la incorporación de materiales cementicios suplementarios en la mezcla que, en general, son subproductos de procesos industriales. Su empleo es deseable no exclusivamente desde el punto de vista de la conservación del medio ambiente y de la energía, sino también por los beneficios que pueden ofrecer al hormigón. Se los puede utilizar como adición o como sustitución parcial del cemento Portland. Algunos ejemplos de materiales cementicios suplementarios son las cenizas volantes, la escoria granulada de alto horno, el humo de sílice y las puzolanas naturales. Específicamente, las cenizas volantes (Fly Ash, FA) se obtienen como residuo de la combustión del carbón en plantas generadoras de electricidad.

Finalmente, el otro tipo de compuesto que se presenta en este trabajo es el hormigón reforzado con fibras, concretamente, con fibras de acero provenientes del reciclado de neumáticos de desecho. En este sentido, es ampliamente conocido que el hormigón se caracteriza por poseer una considerable resistencia a la compresión, sin embargo, cuando dominan los mecanismos de falla por tracción presenta muy baja resistencia y ductilidad. Por tanto, incrementar la tenacidad del hormigón conduciría a mejorar su comportamiento resistente global. De hecho, agregar una pequeña fracción (generalmente del orden de $0,5 \%$ al $1,0 \%$ en volumen) de fibras cortas es una forma efectiva de mejorar la tenacidad del compuesto y obtener un material al que, en general, se lo denomina compuesto cementicio reforzado con fibras (fiber-reinforced cementitious composite, FRCC) o, en particular, hormigón reforzado con fibras ( $f$ iber-reinforced concrete, FRC). En cuanto al tipo de fibras, se han utilizado de diversos materiales tales como acero, vidrio, celulosa natural, carbono, 
polipropileno, entre otros (ACl-544.1-96, 1996). A propósito de los LCC, resulta atractiva la reutilización de las fibras de acero provenientes de neumáticos usados, ya que su eliminación se ha convertido en un gran problema en la gestión de residuos y su creciente cantidad constituye una grave amenaza para el medio ambiente y la salud humana. En efecto, la Directiva del Consejo 1999/31/CE de la Comisión Europea sobre rellenos sanitarios de residuos (EU-Directive 1999/31/EC, 1999) estableció que, desde 2003, ya no pueden ser dispuestos en ellos los neumáticos enteros usados y, a partir de 2006, tampoco en estado triturado. Por consiguiente, existe una fuerte motivación para reciclar estos residuos ya que pueden convertirse en una fuente de materias primas secundarias: las fibras recicladas obtenidas del refuerzo interno de acero se pueden usar en sustitución de las fibras industriales para producir FRC ecosustentable.

En síntesis, en este artículo se describen las actividades experimentales realizadas sobre el estudio del comportamiento de los LCC, que fueron desarrolladas en el marco de los proyectos de investigación denominados "Environmentally-friendly solutions for Concrete with Recycled and natural components" (EnCoRe, FP7-PEOPLE-IRSES-2011) $y$ "SUstainability-driven international/intersectoral Partnership for Education and Research in modeIling next generation CONCRETE" (SUPERCONCRETE, H2020-MSCA-RISE-2014).

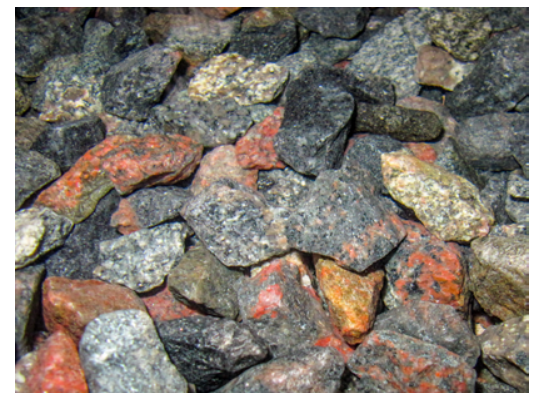

(a)

\section{METODOLOGÍA}

En esta sección se describen los materiales utilizados y los procedimientos experimentales Ilevados a cabo para cada tipo de LCC analizado.

\section{Agregados reciclados en el hormigón}

El programa experimental desarrollado en la Facultad de Ingeniería de la Universidad de Buenos Aires para caracterizar mecánicamente a los RAC incluyó, entre otros, ensayos a compresión uniaxial y triaxial con tres niveles de confinamiento. Inicialmente, se diseñó y elaboró un hormigón de referencia con una resistencia a la compresión de 35 MPa. Se utilizaron agregados gruesos triturados de origen granítico y con tamaño máximo nominal de $19 \mathrm{~mm}$, y como agregados finos, una combinación de arena fina de río y arena de trituración. Una vez finalizados los ensayos mecánicos previstos sobre las probetas elaboradas con este hormigón, se trituraron los especímenes, y los agregados gruesos obtenidos de este proceso fueron posteriormente reutilizados para la producción de RAC con la misma dosificación original (figura 1). Se descartaron todos los agregados reciclados más finos que el tamiz de 4,75 $\mathrm{mm}$ de abertura (Palacio-León, Chávez-Porras y Velásquez-Castiblanco, 2017). Adicionalmente, se consideraron tres porcentajes de reemplazo $(R)$ de agregados gruesos

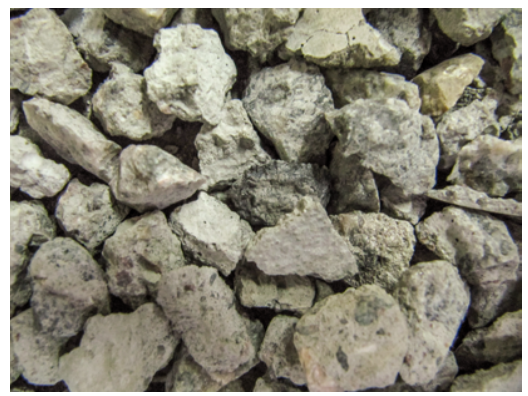

(b)

Figura 1. (a) Agregados gruesos graníticos usados para el hormigón de referencia y (b) RCA obtenido luego del proceso de trituración del mismo hormigón

Fuente: elaboración propia. 
naturales por reciclados. En este sentido, se denominó NAC al hormigón sin agregados reciclados, y R30 \%, R60 \% y R100 \% a los RAC con un valor $\mathrm{R}$ de $30 \%$, $60 \%$ y $100 \%$, respectivamente. En la tabla 1 se aprecia la disminución tanto de la consistencia como de la densidad de las mezclas en estado fresco a medida que se incrementa el nivel de reemplazo R (Folino y Xargay, 2014).

\section{Reemplazo de cemento Portland por cenizas volantes}

La actividad descrita en este parágrafo se desarroIló en la Universitá degli Studi di Salerno y tuvo por objetivo profundizar el conocimiento sobre el comportamiento mecánico y la durabilidad de los hormigones fabricados con RCA y FA, y su posible aplicación con fines estructurales. Para ello, a partir de una composición de hormigón de referencia se diseñaron 12 mezclas, reemplazando parte de los constituyentes ordinarios (es decir, cemento, arena y agregados gruesos) por FA y RCA. Algunas de las características mecánicas evaluadas fueron la resistencia a la compresión y la resistencia a la tracción indirecta, mientras que la durabilidad se examinó mediante la determinación de la permeabilidad al agua, la profundidad de carbonatación y el ingreso de iones de cloruro (Martinelli, Koenders y Caggiano, 2013; Lima et al., 2013; Pepe, Koenders, Faella y Martinelli, 2014).

Las tablas 2 y 3 brindan información fundamental de los agregados empleados en la campaña.

Tabla 1. Resultados de ensayos físicos en estado fresco de las mezclas estudiadas

\begin{tabular}{lcccc}
\hline & NAC & R30 \% & R60 \% & R100 \% \\
\hline Asentamiento $[\mathrm{mm}]$ & 180 & 170 & 80 & 55 \\
\hline Densidad $\left[\mathrm{kg} / \mathrm{m}^{3}\right]$ & 2420 & 2385 & 2382 & 2346 \\
\hline
\end{tabular}

Fuente: elaboración propia.

Tabla 2. Aspecto de los agregados naturales y reciclados clasificados según sus fracciones granulométricas

\begin{tabular}{lllll}
\hline $\begin{array}{c}\text { Tipo de } \\
\text { agregado }\end{array}$ & Arena (0-2mm) & N1 (2-10mm) & N2 (10-20mm) & N3 (20-31,5mm) \\
Naturales & & & \\
\hline & & & \\
Reciclados & & & \\
\hline
\end{tabular}

Fuente: elaboración propia.

Tabla 3. Características físicas de los agregados

\begin{tabular}{lccccc}
\hline \multirow{2}{*}{ Tipo } & Densidad & \multicolumn{4}{c}{ Absorción de agua [\%] } \\
\cline { 3 - 6 } & {$\left[\mathbf{k g} / \mathbf{m}^{\mathbf{3}}\right]$} & Arena (0-2mm) & N1 (2-10mm) & N2 (10-20mm) & N3 (20-31,5mm) \\
\hline Natural & 2690 & 1,20 & 0,70 & 0,50 & 0,30 \\
\hline Reciclado & 2369 & 12,20 & 6,00 & 3,00 & 1,80 \\
\hline
\end{tabular}

Fuente: elaboración propia. 
Además, la primera columna de la tabla 4 informa un código que denota la mezcla y proporciona datos clave sobre el tipo de agregados y el contenido de cenizas volantes.

En particular, el agregado natural se denota con la letra $\mathrm{N}$; mientras que con $\mathrm{R}$ se indica la presencia de un porcentaje dado de agregados reciclados (30, 60 o $100 \%$ ). Adicionalmente, una primera letra indica el nivel de contenido de cenizas volantes $\left(\mathrm{L}\right.$, para contenido bajo de $80 \mathrm{~kg} / \mathrm{m}^{3} ; \mathrm{M}$, para medio de $220 \mathrm{~kg} / \mathrm{m}^{3}$, y $\mathrm{H}$, para alto de $255 \mathrm{~kg} /$ $\mathrm{m}^{3}$ ). La mezcla de referencia fue la $N$, diseñada únicamente con agregados naturales según EN 206-1 (2006) para una clase de exposición XC2 y de resistencia C25/30. Para esta mezcla de referencia se utilizó la cantidad mínima de cemento sugerida por la norma europea mencionada, igual a $280 \mathrm{~kg} / \mathrm{m}^{3}$. También de la tabla 4, se puede observar que la densidad de los RAC fue siempre menor que la de los hormigones elaborados con agregados naturales. Esto se debe a que los RCA poseen mayor porosidad y, por ende, menor densidad que los naturales (ídem tabla 1 ).

\section{Incorporación de fibras de acero recicladas en el hormigón}

A continuación, se describen las acciones realizadas con el propósito de estudiar el comportamiento mecánico de FRC elaborado con fibras de acero industriales y recicladas, estas últimas recuperadas de neumáticos de desecho. Para este fin, se prepararon especímenes de diferentes mezclas, caracterizadas por contener un mismo volumen de fibras pero en diferentes proporciones de fibras industriales y recicladas, y fueron evaluados tanto a compresión como a flexión. Más específicamente, se partió de una mezcla de FRC de referencia con $0,5 \%$ en volumen de fibras de acero industriales (industrial steel fibers, ISF), es decir $40 \mathrm{~kg} /$ $\mathrm{m}^{3}$, y se prepararon tres mezclas más reemplazando el $25 \%$, $50 \%$ y $100 \%$ de dichas fibras con una cantidad igual de fibras recicladas (recycled steel fibers, RSF). De este modo, el comportamiento mecánico del hormigón reforzado solo con ISF fue comparado con el hormigón con un híbrido de fibras de acero (industriales y recicladas) y

Tabla 4. Composición de las mezclas evaluadas

\begin{tabular}{ccccccccc}
\hline Mezcla & $\begin{array}{c}\text { Densidad } \\
{\left[\mathbf{k g} / \mathbf{m}^{3}\right]}\end{array}$ & $\begin{array}{c}\text { NA } \\
{[\mathbf{\%}]}\end{array}$ & $\begin{array}{c}\mathbf{N A} \\
{\left[\mathbf{k g} / \mathbf{m}^{3}\right]}\end{array}$ & $\begin{array}{c}\mathbf{R C A} \\
{[\mathbf{\%}]}\end{array}$ & $\begin{array}{c}\mathbf{R C A} \\
{\left[\mathbf{k g} / \mathbf{m}^{3}\right]}\end{array}$ & $\begin{array}{c}\text { Agua } \\
{\left[\mathbf{k g} / \mathbf{m}^{3}\right]}\end{array}$ & $\begin{array}{c}\mathbf{F l y ~ A s h} \\
{\left[\mathbf{k g} / \mathbf{m}^{3}\right]}\end{array}$ & $\begin{array}{c}\text { CEM I 42,5R } \\
{\left[\mathbf{k g} / \mathbf{m}^{3}\right]}\end{array}$ \\
\hline $\mathrm{N}$ & 2414 & 100 & 1970 & 0 & 0 & 164,0 & - & 280 \\
\hline $\mathrm{LN}$ & 2384 & 100 & 1890 & 0 & 0 & 164,0 & 80 & 250 \\
\hline LR30 & 2325 & 70 & 1323 & 30 & 500 & 171,9 & 80 & 250 \\
\hline LR60 & 2273 & 40 & 750 & 60 & 1005 & 188,2 & 80 & 250 \\
\hline LR100 & 2255 & 0 & 0 & 100 & 1665 & 259,6 & 80 & 250 \\
\hline MN & 2336 & 100 & 1705 & 0 & 0 & 161,3 & 220 & 250 \\
\hline MR30 & 2283 & 70 & 1195 & 30 & 450 & 167,8 & 220 & 250 \\
\hline MR60 & 2239 & 40 & 685 & 60 & 905 & 179,0 & 220 & 250 \\
\hline MR100 & 2219 & 0 & 0 & 100 & 1500 & 248,8 & 220 & 250 \\
\hline HN & 2321 & 100 & 1705 & 0 & 0 & 161,3 & 255 & 200 \\
\hline HR30 & 2268 & 70 & 1195 & 30 & 450 & 167,8 & 255 & 200 \\
\hline HR60 & 2209 & 40 & 675 & 60 & 900 & 178,7 & 255 & 200 \\
\hline HR100 & 2204 & 0 & 0 & 100 & 1500 & 248,8 & 255 & 200 \\
\hline
\end{tabular}

Fuente: elaboración propia. 
también con el hormigón reforzado solo con RSF, con el objetivo de determinar la factibilidad de su uso como material estructural (Caggiano, Xargay y Martinelli, 2015; Caggiano et al., 2017; Martinelli, Caggiano y Xargay, 2015).

Las fibras de acero recicladas empleadas en este estudio (figura 2a) fueron suministradas por una empresa dedicada a recolectar y reciclar neumáticos usados. Se realizó una caracterización geométrica detallada en un grupo de aproximadamente 2000 RSF muestreadas al azar. De acuerdo con dichas mediciones, el diámetro promedio de las fibras oscilaba entre 0,11 y 1,64 mm, caracterizado por un valor medio de $0,27 \mathrm{~mm}$. El valor medio de la proyección de la longitud de las fibras fue de 12 mm y el $47 \%$ de las longitudes de fibra medidas se

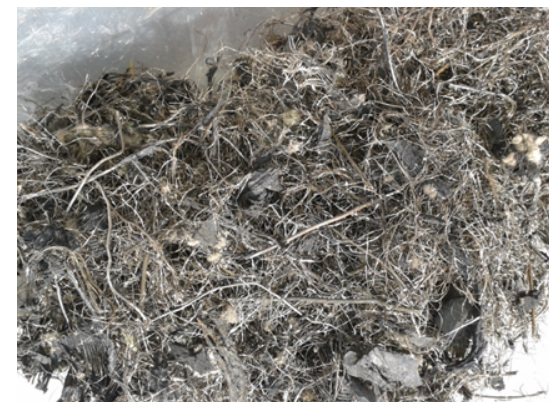

(a)

Figura 2. Fibras de acero (a) recicladas e (b) industriales

Fuente: elaboración propia.

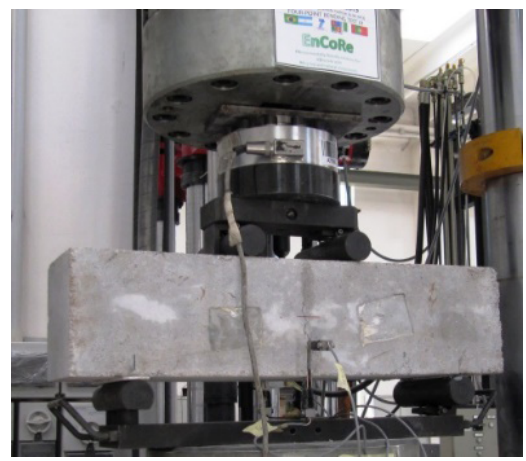

encontró entre 9 y $15 \mathrm{~mm}$. Por otra parte, en este estudio se consideraron las fibras industriales Wirand FS7 con ganchos en los extremos (figura 2b), que en lo siguiente serán genéricamente referidas como ISF. Sus principales propiedades geométricas y mecánicas son: longitud de la fibra $33 \mathrm{~mm}$, diámetro de la fibra 0,55 mm, relación de esbeltez (longitud característica/diámetro) 60 y resistencia a la tracción 1200 MPa.

Se realizaron ensayos a tracción por flexión en cuatro puntos con el objetivo de caracterizar el comportamiento posfisuración de las muestras de FRC. Estas pruebas se ejecutaron de acuerdo con lo establecido por la norma (UNI-11039-2, 2003). En la figura 3 se puede apreciar el esquema experimental con la instrumentación de un espécimen.

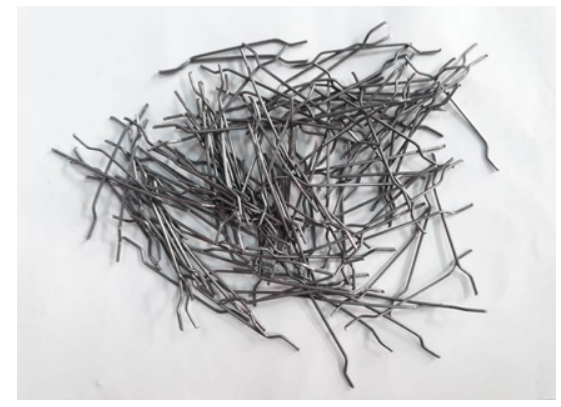

(b)

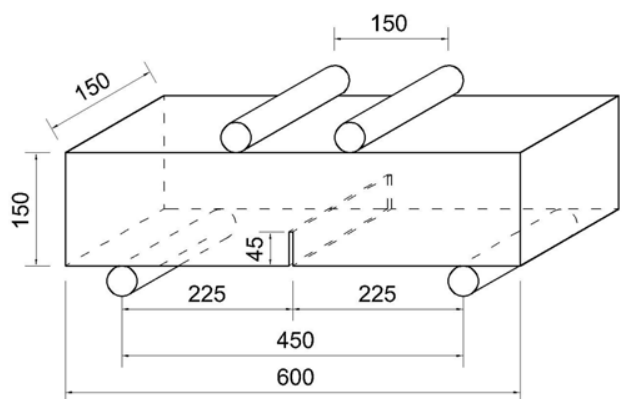

Figura 3. Configuración general del ensayo a flexión en cuatro puntos con viga entallada

Fuente: elaboración propia. 


\section{RESULTADOS}

A continuación, se presentan separadamente los principales resultados alcanzados en el desarrollo de las campañas experimentales.

\section{Agregados reciclados en el hormigón}

En este apartado, se resumen los resultados obtenidos de la evaluación mecánica de los hormigones elaborados con agregados gruesos reciclados. En la figura 4 se presentan las curvas tensión vs deformación del ensayo de compresión uniaxial, donde puede observarse que tanto la resistencia a compresión $\left(\sigma_{3}\right)$ como el módulo de Young (E) disminuyeron al aumentar el reemplazo $\mathrm{R}$ de agregados. En la figura 5 se resumen los resultados de las pruebas triaxiales, donde se aprecia que el confinamiento mejoró considerablemente la resistencia a la compresión y que con el incremento de $\mathrm{R}$ el hormigón fue ligeramente más sensible al confinamiento. En resumen, el uso de hormigón triturado como reemplazo de los agregados de origen natural modificó las propiedades

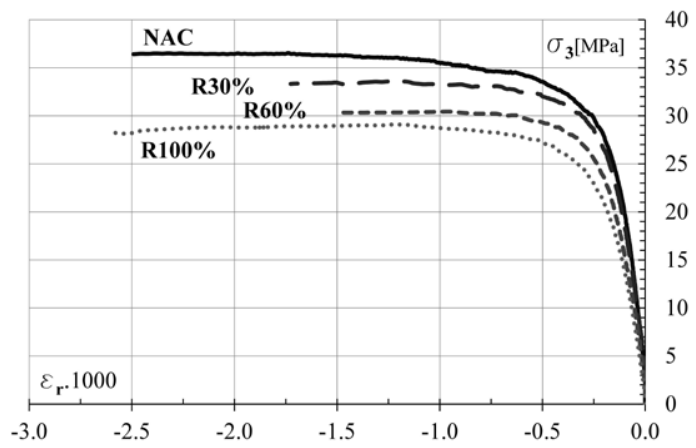

(b)

(a)

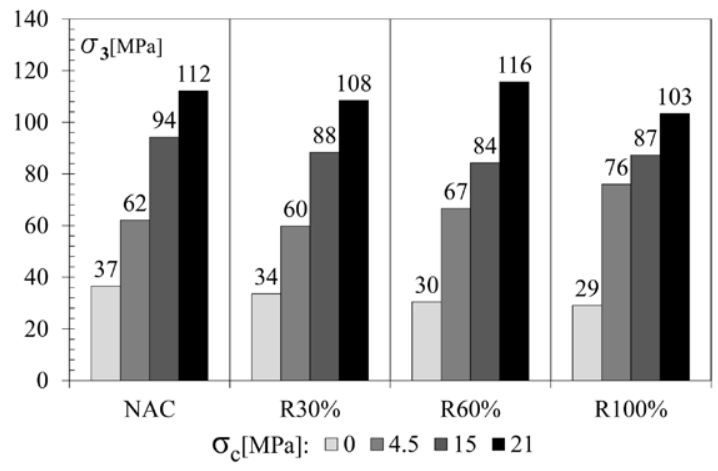

(b)

Figura 5. Resultados de compresión triaxial en RAC: (a) curvas medias completas de tensión $\sigma_{3}$ vs. deformación axial $\varepsilon_{a}$ para R30 \% y R60 \%; (b) evolución de tensión pico $\sigma_{3}$ según el confinamiento $\sigma_{c}$ aplicado

Fuente: elaboración propia. 
fundamentales de la mezcla de referencia. Se debe destacar la degradación de su resistencia y rigidez debido a la mayor porosidad y capacidad de absorción de agua de los agregados reciclados.

\section{Reemplazo de cemento Portland por cenizas volantes}

En cuanto a la incorporación de cenizas volantes en la mezcla cementicia, se estudió su incidencia en las propiedades físico-mecánicas del hormigón resultante. En la figura 6 se muestran los resultados de resistencia a compresión uniaxial para cada tipo de mezcla a distintas edades. Se puede observar que la sustitución del agregado natural por los reciclados disminuyó la resistencia a la compresión para cada edad de curado. No obstante, la sustitución parcial del cemento Portland por FA generó un incremento de la resistencia para edades mayores a 60 días.

En la figura 7 se puede apreciar la evolución de la resistencia a compresión uniaxial en función del porcentaje de reemplazo de agregados reciclados, para distintos contenidos de cenizas volantes. Se distingue claramente la influencia simultánea del uso de distintos porcentajes de RCA y de FA, sobre la resistencia a compresión uniaxial del RAC. En dicha figura, se muestra el valor adimensional $\mathrm{f}_{\mathrm{c}^{\prime}} \mathrm{f}_{{ }^{\prime}}{ }_{\mathrm{c}^{\prime}}$ siendo este la relación entre la resistencia a

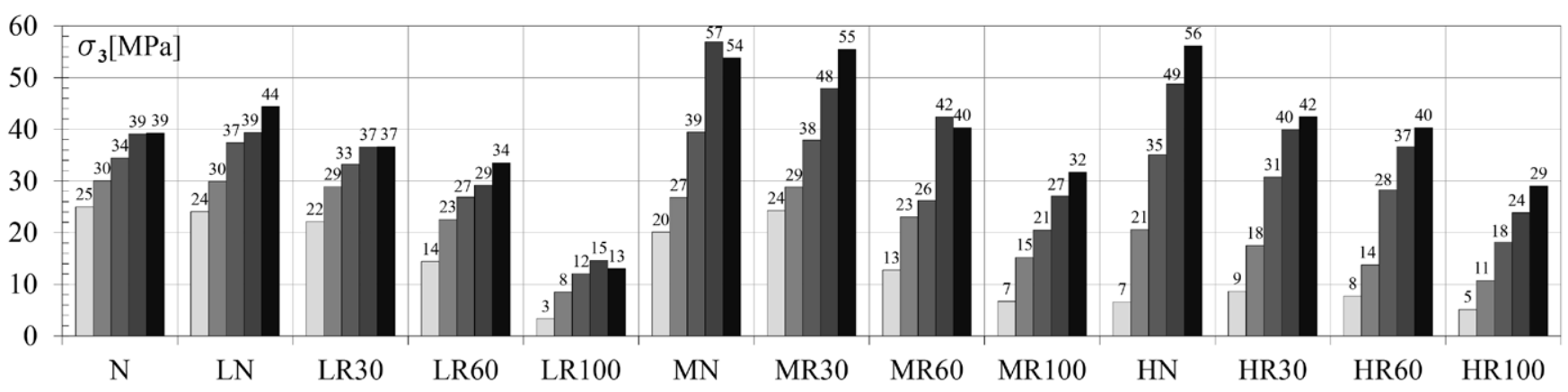

Figura 6. Variación temporal de la resistencia a compresión uniaxial

Fuente: elaboración propia.
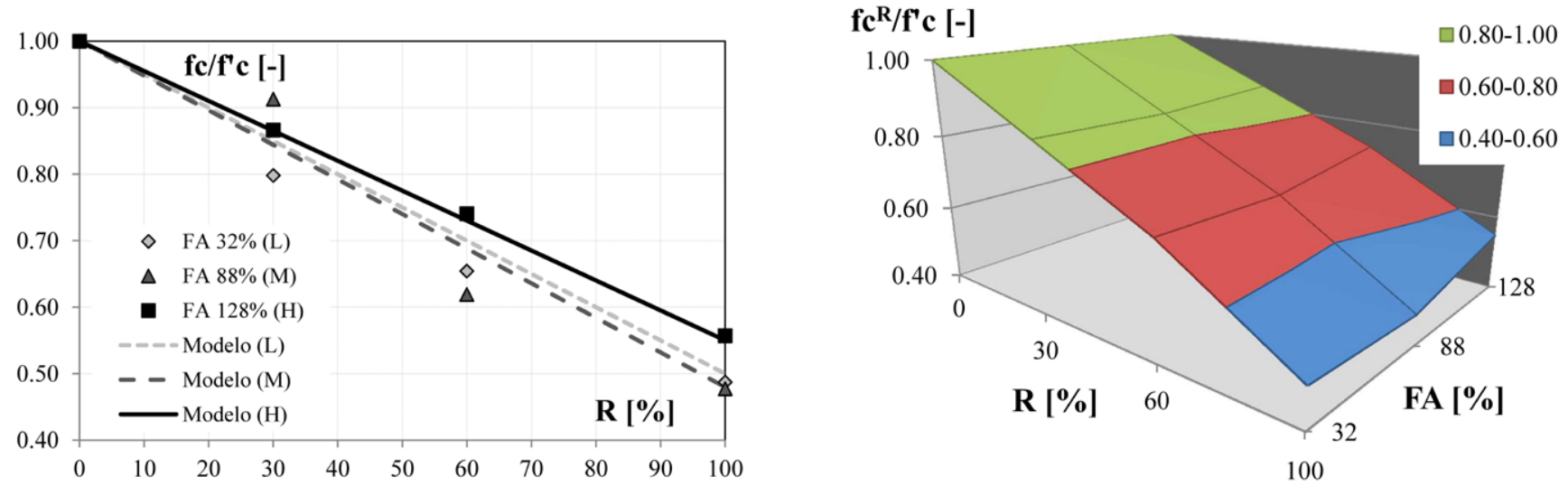

Figura 7. Evolución de la resistencia a compresión uniaxial $f_{c}$ para distintos porcentajes de FA y $R$

Fuente: elaboración propia. 
compresión $\left(f_{c}\right)$ de las mezclas con componentes reciclados, respecto al valor de la resistencia a compresión de la mezcla patrón $\left(\mathrm{f}_{\mathrm{c}}{ }_{\mathrm{c}}\right)$. Asimismo, los valores experimentales $f_{c} / f^{\prime}{ }_{c}$ se detallan en la tabla 5 . Puede observarse que conforme aumenta el contenido de RCA, disminuye $f_{c}$. Contrariamente, la resistencia a compresión uniaxial aumenta con el contenido de FA. El comportamiento mecánico previamente descrito puede ajustarse con suficiente exactitud a través de relaciones lineales, como se muestra en la figura 7 . En la tabla 5 se presentan las pendientes de esas rectas, Ilamadas $\alpha_{R^{\prime}}$ calibradas a partir de los resultados experimentales para un valor FA dado. Cuanto mayor es el contenido de FA, menor resulta $\alpha_{R}$.

Tabla 5. Relaciones $f_{c} / f^{\prime}{ }_{c}$ y pendientes $a_{R}$ para distintos contenidos de FA y RCA.

\begin{tabular}{|c|c|c|c|}
\hline \multirow[b]{2}{*}{$\mathbf{R}[\%]$} & \multicolumn{3}{|c|}{ Resistencia a compresión relativa $\left(\mathrm{f}_{\mathrm{c}} / \mathbf{f}_{\mathrm{c}}^{\prime}\right)$} \\
\hline & $\begin{array}{c}\text { FA 32 \% (L) } \\
\alpha_{R}=5,0 \% 0\end{array}$ & $\begin{array}{c}\text { FA 88 } \%(M) \\
\alpha_{R}=5,2 \% 0\end{array}$ & $\begin{array}{c}\text { FA } 128 \%(\mathrm{H}) \\
\alpha_{\mathrm{R}}=4,5 \% \mathrm{o}\end{array}$ \\
\hline 0 & 1,00 & 1,00 & 1,00 \\
\hline 30 & 0,85 & 0,84 & 0,87 \\
\hline 60 & 0,70 & 0,69 & 0,73 \\
\hline 100 & 0,50 & 0,48 & 0,55 \\
\hline
\end{tabular}

Fuente: elaboración propia.

En lo que respecta a las características asociadas a la durabilidad, como es conocido, la permeabilidad del hormigón se rige principalmente por las características de la pasta más que por las de los agregados. Por tanto, la presencia de FA en el hormigón redujo la permeabilidad del material compuesto debido a que refina la microestructura de la pasta, compensando el aumento esperado de la porosidad por la presencia de RCA. Las mezclas de hormigón con cantidades más altas de FA mostraron la menor permeabilidad al agua. Por su parte, la penetración de iones de cloruro se ve incrementada por la presencia de RCA en el hormigón, pero, al mismo tiempo, el uso de FA la disminuye. La profundidad de la carbonatación se controla principalmente por las características de la pasta: es mayor para valores más altos de la relación efectiva de agua/aglomerantes.

Los resultados destacaron que el comportamiento mecánico y de durabilidad más deficiente del hormigón producido con agregados reciclados -inducido por la alta porosidad de los RCA- se puede correlacionar con la mayor proporción de relación agua/aglomerantes que se deriva de los procedimientos estándar adoptados para la dosificación de hormigón estructural. Además, el aumento porcentual de los agregados reciclados ha sido perjudicial en términos de trabajabilidad del hormigón en estado fresco, aun incorporando mayores cantidades de aditivos fluidificantes. También se han investigado muestras de hormigón con contenidos de cemento menores y con cenizas volantes mayores que los límites establecidos por las actuales normas europeas. Si bien estos hormigones han mostrado propiedades mecánicas y físicas más pobres, aún es factible aceptar una ampliación de dichos límites y debería implementarse en las futuras versiones de los estándares mundiales, como un impulso adicional hacia la producción de hormigones estructurales más sostenibles. En síntesis, los resultados obtenidos revelan que el uso combinado RCA y FA puede lograr un efecto sinérgico en términos de las propiedades físicas y mecánicas más relevantes del hormigón, mitigando las consecuencias desfavorables del uso de estos agregados. Las mezclas producidas con incluso una gran cantidad de RCA y FA muestran resultados aceptables para su aplicación como hormigón estructural.

\section{Incorporación de fibras de acero recicladas en el hormigón}

A continuación se detallan los resultados de los ensayos mecánicos realizados en hormigones reforzados con fibras de acero recicladas. En la tabla 6 se resumen los valores pico medios obtenidos de las pruebas a compresión en muestras cúbicas de hormigón simple (PC) y de FRC. No se observaron diferencias significativas entre los especímenes sin refuerzo y los de FRC, esto significa que dicha 
resistencia se encuentra principalmente controlada por las propiedades de la matriz cementicia.

Tabla 6. Resultados de los ensayos a compresión uniaxial en cubos $f^{\prime}$ y densidades

\begin{tabular}{lcccc}
\hline \multirow{2}{*}{ Mezcla } & \multicolumn{2}{c}{$\begin{array}{c}\text { Densidad } \\
{\left[\mathbf{k g} / \mathbf{m}^{3}\right]}\end{array}$} & \multicolumn{2}{c}{$\mathbf{f}^{\prime} \mathbf{c}$ a 28 días } \\
& {$[\mathbf{M P a}]$} \\
\cline { 2 - 5 } & $\mathbf{P C}$ & SFRC & PC & SFRC \\
\hline REFERENCIA & 2371 & & 42,59 & \\
\hline RSFRC 0-05 & 2376 & 2413 & 40,57 & 39,01 \\
\hline RSFRC 25-05 & 2428 & 2435 & 36,42 & 36,52 \\
\hline RSFRC 50-05 & 2459 & 2450 & 36,89 & 36,74 \\
\hline RSFRC 100-05 & 2446 & 2491 & 36,69 & 37,37 \\
\hline
\end{tabular}

Fuente: elaboración propia.
La figura 8 muestra las curvas experimentales de la carga vertical $(P)$ en función de los desplazamientos de abertura de la punta de fisura (CTODm, mean value of two Crack Tip Opening Displacements measured) registrados en los ensayos a tracción por flexión en cuatro puntos. En la figura 9 se observa el aspecto de las superficies completamente fracturadas de las vigas y la distribución de las fibras, luego de finalizar los ensayos a flexión. Por su parte, en la figura 10 se presentan comparativamente tres parámetros característicos establecidos por la norma (UNI-11039-2, 2003), que describen la respuesta del material compuesto luego de la fisuración. Estos se definen como la resistencia a la primera fisuración $\left(\mathfrak{f}_{\mid f}\right)$ y dos resistencias

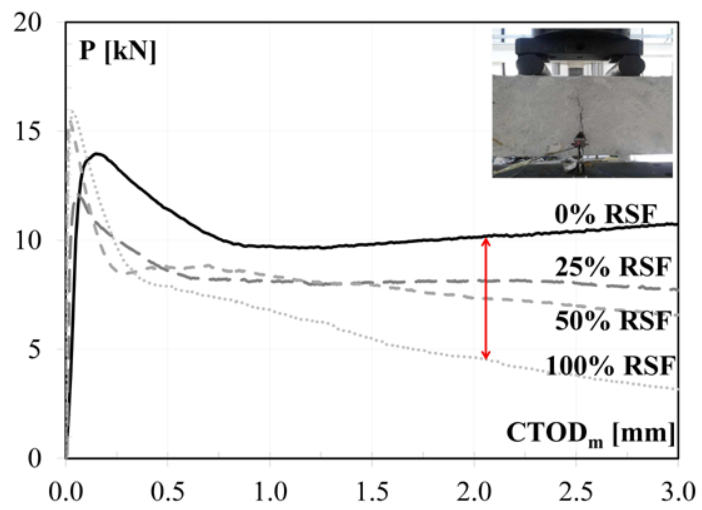

Figura 8. Resultados de ensayos a flexión en cuatro puntos: curvas de carga vertical P vs. CTODm

Fuente: elaboración propia.
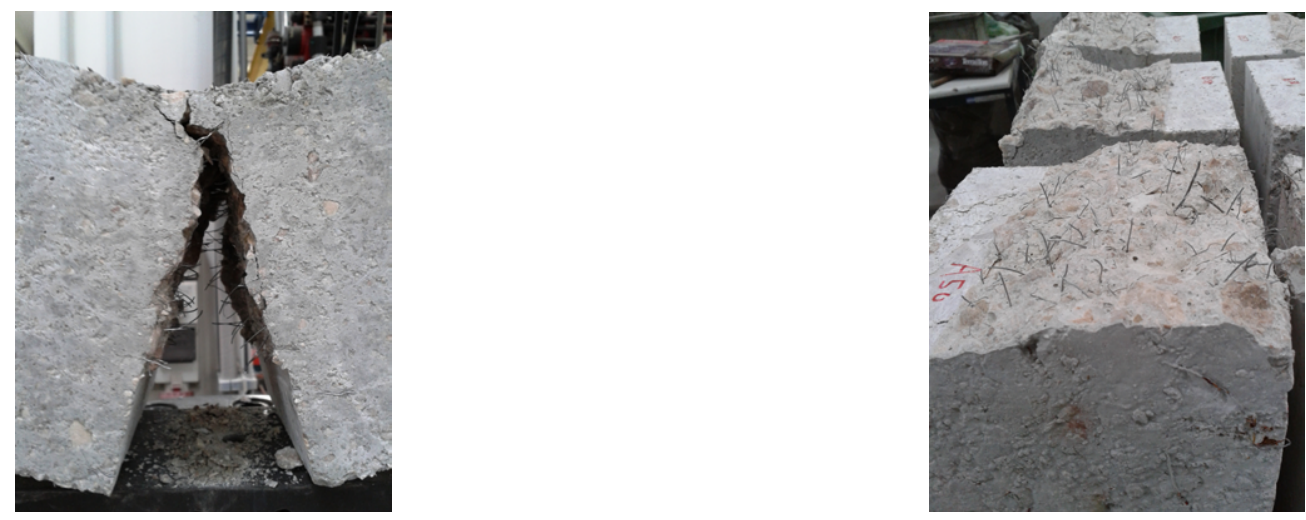

Figura 9. Detalle de distribución de fibras en especímenes luego de ser ensayados

Fuente: elaboración propia. 


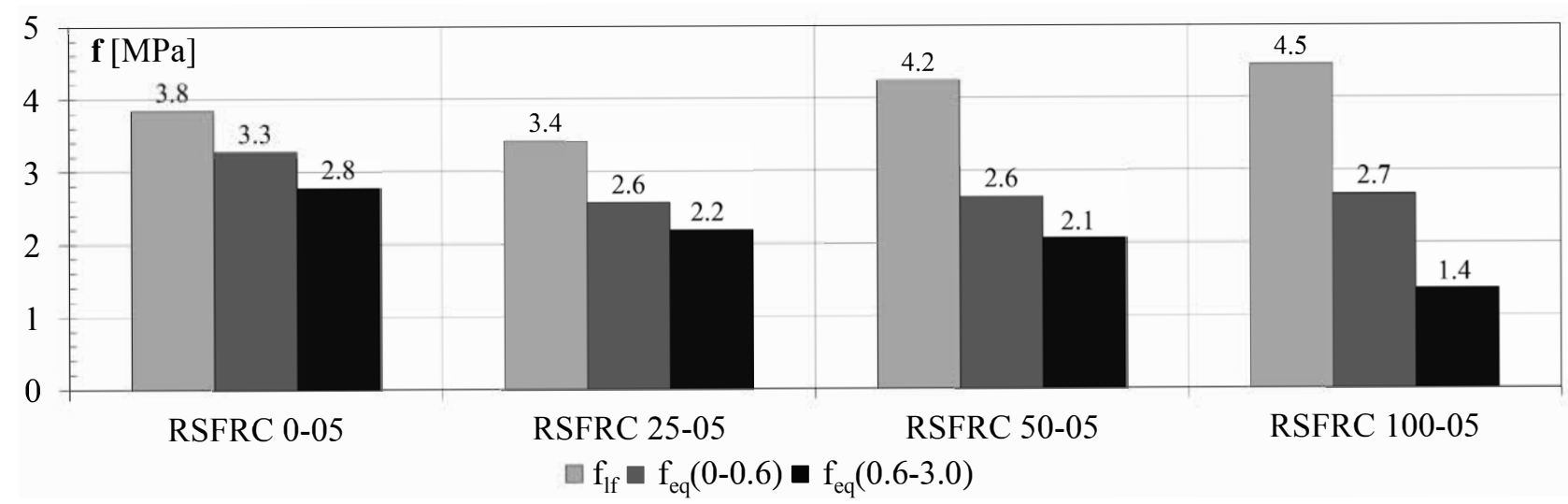

Figura 10. Parámetros característicos medios del ensayo a flexión en cuatro puntos: resistencia a la primera fisuración, $f_{\text {If }^{\prime}}$ y resistencias equivalentes posfisuración, $f_{\text {eq }}(0-0.6)$ y $f_{\text {eq }}(0.6-3.0)$

Fuente: elaboración propia.

equivalentes post-fisuración: la primera $\mathrm{f}_{\text {eq(0-0.6) }} \mathrm{CO}-$ responde a un CTOD que varía entre $\mathrm{CTOD}_{0}$ y $\mathrm{CTOD}_{0}+0,6 \mathrm{~mm}$ y es relevante para el estado límite de servicio, mientras que el segundo $f_{\text {eq }(0,6-3,0)}$ se refiere a un CTOD que varía entre $\mathrm{CTOD}_{0}+0,6$ y $\mathrm{CTOD}_{0}+3,0 \mathrm{~mm}$, y es representativo del estado límite último. $\mathrm{CTOD}_{0}$ representa el CTOD correspondiente a la carga máxima de la muestra de hormigón simple, es decir, sin fibras.

El efecto del reemplazo de ISF por RSF se puede comprender mediante el análisis las curvas presentadas. La respuesta posfisuración de los especímenes FRC reforzados solo con ISF se caracterizó por una tenacidad y ductilidad muy significativa. En cambio, las muestras con una mayor cantidad de RSF en sustitución de ISF mostraron generalmente una etapa de ablandamiento más pronunciada luego del pico de carga. Esto señala una eficiencia menor de las fibras recicladas con respecto a las industriales, que están diseñadas específicamente para mostrar una excelente interacción con la matriz. En particular, las fibras recicladas no son rectas, no tienen ganchos de anclaje y tienen relaciones de esbeltez más bajas.

Los resultados confirmaron la prometedora aplicación del hormigón reforzado con fibras de acero recicladas de neumáticos usados. Las propiedades resultantes superaron los valores mínimos reglamentarios exigidos para su uso como material estructural. Sin embargo, la evidencia experimental demuestra que las fibras industriales no pueden ser reemplazadas por una cantidad igual de fibras recicladas sin derivar en un deterioro de la respuesta a la tracción pospico del compuesto.

\section{CONCLUSIONES}

En este trabajo, se presentó una síntesis de las actividades científicas realizadas en el contexto de los proyectos EnCoRe y SUPERCONCRETE, que trataron el concepto, la caracterización material y aplicación estructural de compuestos ecosustentables, elaborados con diversos tipos de componentes reciclados o naturales. Los proyectos han abarcado desde el uso clásico de materias primas secundarias como sustitutos del cemento, por ejemplo, cenizas volantes, así como también consolidar tecnologías emergentes, como el uso de agregados de hormigón reciclado de residuos de demolición y fibras de acero del reciclado de neumáticos. Se han identificado novedosas líneas de investigación en los efectos sinérgicos provocados por el uso simultáneo de más de un componente reciclado.

Por último y como corolario de las actividades desarrolladas, se ha establecido y difundido una base conceptual científica para el uso de estos 
materiales verdes y tecnologías asociadas en el sector de la construcción. Esta ha sido una exitosa experiencia internacional, basada en el intercambio académico, donde la investigación aplicada en ingeniería hizo su aporte específico para impulsar un desarrollo económico y social sostenible. En el futuro se deberán intensificar los esfuerzos dirigidos a incrementar la sustentabilidad de la industria de la construcción, con énfasis en ampliar las regulaciones existentes para incorporar estos nuevos materiales. De hecho, este marco regula inevitablemente la posibilidad de expandir su uso en los sectores de la construcción y la ingeniería civil.

\section{FINANCIAMIENTO}

Las investigaciones presentadas en este trabajo se desarrollaron en el marco de los proyectos EnCoRe (FP7-PEOPLE-2011-IRSES, No. 295283, 20122014, https://cordis.europa.eu/project/rcn/102094/ factsheet/es) y SUPERCONCRETE (H2020-MSCA-RISE-2014, No.645704, 2015-2019, https:// cordis.europa.eu/project/rcn/194367/es) financiados por la Comisión Europea.

\section{REFERENCIAS}

121-DRG RILEM TC (1994). Specification for concrete with recycled aggregates. Materials and Structures, 27(173), 557-559.

ACI 555-R01 (2001). Removal and reuse of hardened concrete. Reporte del ACI Committee 555. Farmington Hills, MI: American Concrete Institute.

ACl-544.1-96 (1996). State-of-the-art report on fiber reinforced concrete. Reporte del ACl Committee 544. Farmington Hills, MI: American Concrete Institute.

Barros, J., Ferrara, L. y Martinelli, E. (2017). Recent Advances on Green Concrete for Structural Purposes. The Contribution of the EU-FP7 Project EnCoRe. Milano: Springer.

Caggiano, A., Folino, P., Lima, C., Martinelli, E. y Pepe, M. (2017). On the mechanical response of Hybrid
Fiber Reinforced Concrete with Recycled and Industrial Steel Fibers. Construction and Building Materials, 147, 286-295.

https://doi.org/10.1016/j.conbuildmat.2017.04.160

Caggiano, A., Xargay, H. y Martinelli, E. (2015). Experimental and numerical characterization of the bond behavior of steel fibers recovered from waste tires embedded in cementitious matrices. Cem. Concr. Compos., 62, 146-155.

https://doi.org/10.1016/j.cemconcomp.2015.04.015

EU-Directive 1999/31/EC. (1999). Council of 26 April 1999 on the landfill of waste (pp. 1-19). Off. J. Eur. Union L182.

Folino, P. y Xargay, H. (2014). Recycled aggregate concrete - Mechanical behavior under uniaxial and triaxial compression. Construction and Building Materials, 56, 21-31.

https://doi.org/10.1016/j.conbuildmat.2014.01.073

Gursel, A., Masanet, E., Horvath, A. y Stadel, A. (2014). Life-cycle inventory analysis of concrete production: a critical review. Cement and Concrete Composites, 51, 38-48.

https://doi.org/10.1016/j.cemconcomp.2014.03.005

Lima, C., Caggiano, A., Faella, C., Martinelli E., M.P. y Realfonzo, R. (2013). Physical properties and mechanical behavior of concrete made with recycled aggregates and fly ash. Construction and Building Materials, 47, 547-559.

https://doi.org/10.1016/j.conbuildmat.2013.04.051

Martinelli, E., Caggiano, A. y Xargay, H. (2015). An experimental study on the post-cracking behaviour of Hybrid Industrial/Recycled Steel Fibre-Reinforced Concrete. Construction and Building Materials, 94, 290-298.

https://doi.org/10.1016/j.conbuildmat.2015.07.007

Martinelli, E., Koenders, E. y Caggiano, A. (2013). A numerical recipe for modelling hydration and heat flow in hardening concrete. Cem. Concr. Compos., 40, 48-58.

https://doi.org/10.1016/j.cemconcomp.2013.06.001

Palacio-León, Ó., Chávez-Porras, Á. y Velásquez-Castiblanco, Y.L. (septiembre de 2017). Evaluación y comparación del análisis granulométrico obtenido 
de agregados naturales y reciclados. Tecnura, 21(53), 96-106.

https://doi.org/10.14483/22487638.8195

Pepe, M., Koenders, E., Faella, C. y Martinelli, E. (2014). Structural concrete made with recycled aggregates: Hydration process and compressive strength models. Mech. Res. Commun., 58, 139-145.

https://doi.org/10.1016/j.mechrescom.2014.02.001

Ripani, M., Etse, G. y Vrech, S. (2017). Recycled aggregate concrete: Localized failure assessment in thermodynamically consistent non-local plasticity framework. Computers and Structures, 178, 47-57. https://doi.org/10.1016/j.compstruc.2016.08.007

Robayo-Salazar, R.A., Mattey-Centeno, P.E., Silva-Urrego, Y.F., Burgos-Galindo, D.M. y Arjona, S. Junio de 2015). Los residuos de la construcción y demolición en la ciudad de Cali: un análisis hacia su gestión, manejo y aprovechamiento. Tecnura, 19(44), 157-170.

https://doi.org/10.14483/udistrital.jour. tecnura.2015.2.a12

UNI-11039-2. (2003). Steel fibre reinforced concreteTest method to determine the first crack strength and ductility indexes. Milán, Italia: UNI Editions.

Villegas-Flores, N. y Parapinski dos Santos, A. C. (Diciembre de 2013). Análisis de indicadores para determinar el grado de sostenibilidad en concretos especiales. Tecnura, 17(38), 12-25.

https://doi.org/10.14483/udistrital.jour. tecnura.2013.4.a01

World Commission on Environment and Development (1987). WCED Our common future. Oxford: Oxford University Press. 